Revista de Derecho Público: Teoría y Método

\title{
OBSERVACIONES TEÓRICAS, HISTÓRICAS Y COMPARADAS SOBRE LA INCERTEZA DEL DERECHO*
}

\section{THEORETICAL, HISTORICAL AND COMPARATIVE REFLECTIONS ON THE UNCERTAINTY OF LAW}

\author{
Jean-Bernard Auby \\ Profesor Emérito de Derecho Público \\ Sciences-Po Paris
}

RESUMEN: En este artículo se exponen, sin pretensión de exhaustividad, algunos problemas que plantea la incerteza que rodea al Derecho y trata de darles solución. Para ello se analiza, en primer lugar, el marco teórico del problema desde una perspectiva ontológica. Posteriormente, se da cuenta del desarrollo histórico de la cuestión y de la situación actual. Por último, se proponen varios métodos para combatir el problema de la incerteza del Derecho en función del tipo de sistema jurídico, pues cada uno presenta una distinta permeabilidad frente a aquella. La tesis fundamental del artículo es que, constatado que en los sistemas jurídicos de nuestro entorno existe incerteza en torno al Derecho, es recomendable reducirla allí donde es posible hacerlo. Para tal análisis se parte de la situación en el Derecho francés y se hace referencia a varios ejemplos de Derecho comparado.

PALABRAS CLAVE: incerteza en el Derecho, complejidad normativa creciente, técnica normativa, codificación, seguridad jurídica.

* Traducción del francés original al espańol realizada por Jorge Castillo Abella, investigador predoctoral contratado (FPI-MINECO) en la Universidad Autónoma de Madrid. En la traducción se han respetado las citas de legislación y jurisprudencia utilizadas por el autor para mayor facilidad en su localización. Este trabajo se publicó originalmente bajo el título "Observations théoriques, historiques et comparatives sur l'incertitude du droit" en la Revue tunisienne des sciences juridiques et politiques, núm. 4, 2018-2, p. 9 y ss.

Fecha de finalización del texto original: 27 de abril de 2018. Fecha de finalización de la traducción: 23 de julio de 2019. 
ABSTRACT: This paper sets out some of the problems arising from the uncertainty that surrounds the law and proposes some solutions to those problems. To that end, we analyse firstly the theoretical framework of the problem from an ontological perspective. Secondly, the historic evolution and current state of the question are examined. Lastly, some methods for reducing the uncertainty of the law are put forward based on the type of legal system, as each type has a different vulnerability to it. The main thesis of this paper is that, once the fact is established that in our legal systems uncertainty surrounds the law, it is advisable to reduce it, where possible. The analysis is based on the situation under French law and refers to various examples of comparative law.

KEYWORDS: uncertainty of law, increasing regulatory complexity, regulatory technique, codification, legal certainty.

SUMARIO: 1. INTRODUCCIÓN.-2. A PROPÓSITO DEL ENCUADRAMIENTO TEÓRICO DEL PROBLEMA: 2.1. Hay una parte de incerteza ontológica del Derecho. 2.2. Esto no impide tratar de reducir el grado de incerteza en un sistema y momento dados.-3. A PROPÓSITO DE LA EVOLUCIÓN HISTÓRICA DEL PROBLEMA: 3.1. Modificaciones del sentido y la función de la ley. 3.2. Complejidad creciente de los sistemas normativos.-4. A PROPÓSITO DE LOS MÉTODOS DE TRATAMIENTO DEL PROBLEMA: 4.1. Las variaciones de la vulnerabilidad de los sistemas frente a la incerteza del Derecho. 4.2. Los métodos más comúnmente utilizados para mejorar la certeza de las reglas. 4.3. Algunas indicaciones sobre la situación en el Derecho francés.-5. CONSIDERACIONES FINALES.-6. BIBLIOGRAFÍA

\section{INTRODUCCIÓN}

No puede abordarse el tema que aquí se va a tratar si no es con una cierta prudencia y modestia. No es un tema nuevo, puede incluso que sea tan viejo como el Derecho, lo que significa que toda clase de plumas eruditas ya se han mojado con su tinta.

Por otra parte, también hay que desconfiar de él puesto que se trata de una figura algo impuesta. Es un componente destacado de la cantinela de la crisis del Derecho y es grande el riesgo de depositar sobre él una capa adicional de tópicos que se añadirían a numerosos otros.

Hacemos aquí una apuesta doble. La primera, concentrarnos en las dimensiones contemporáneas del objeto: aunque se trate de una cuestión antigua, el tiempo presente le confiere indiscutiblemente dimensiones particulares que merecen ser puestas de relieve.

La segunda es introducir una dosis de subjetividad. En cierto modo, cada jurista tiene su propia percepción del grado de incerteza del Derecho, pues esta percepción parte fundamentalmente de la experiencia personal: la aproximación al Derecho está influida por el sentimiento que cada uno tiene, o no tiene, de que es una realidad que siempre huye, se desvanece o se aleja de lo que debería ser para ser bien comprendida.

Como veremos, nuestra visión es más bien relativista y debe mucho a las tesis realistas y empíricas. El sentido del Derecho no parece ser nunca evidente y transparente, por razones explicadas por personas más sabias que nosotros y sobre las que 
volveremos brevemente más adelante. Nos parece más bien probable que la incerteza sea una parte esencial del Derecho.

Sin embargo, no por ello es menos necesario reducirla en la medida de lo posible.

Explicaremos esta posición a través de ciertas observaciones relativas al encuadramiento teórico del problema (2), su evolución histórica (3) y los métodos de tratamiento que pueden aplicársele (4).

\section{A PROPÓSITO DEL ENCUADRAMIENTO TEÓRICO DEL PROBLEMA}

Sin profundizar mucho en las reflexiones sobre la naturaleza misma del Derecho, nos parece que se puede establecer que hay una parte de incerteza ontológica del Derecho (2.1), lo que no impide tratar de reducir el nivel de incerteza que se observe en un sistema y momento dados (2.2).

\subsection{Hay una parte de incerteza ontológica del Derecho ${ }^{1}$}

a) Para convencerse de ello debe decirse de entrada que la norma jurídica puede ser incierta por diversas razones y de diversas maneras.

En lo que se piensa de forma espontánea es la incerteza que afecta al sentido, al contenido de la regla: ¿qué significa una cierta disposición legislativa o convencional redactada de manera alambicada?

Pero debe tenerse en cuenta que la incerteza normativa también puede afectar a la validez, el alcance o incluso a la autoridad de la norma. ¿Realmente está esta norma aún en vigor? ¿Constituye esta otra norma una excepción a la solución general, o cede ante esta? ¿Prevalece realmente una norma sobre otra norma concurrente?

A estas podrían añadirse las incertezas que afectan a la efectividad de la regla. Es sabido que ciertas reglas son ignoradas o consideradas derogadas tácitamente (désuètes) por las autoridades que, en principio, se encargan de su aplicación, mientras que otras reglas se sitúan bajo una estrecha vigilancia de los poderes públicos...

b) Si hay un nivel irreductible, ontológico, de incerteza del Derecho es porque en todos estos aspectos pueden juegan factores que hacen dudar del sentido y el efecto de las reglas.

En ocasiones sucede que el autor de la regla no sabe escribir bien, pero si es así normalmente se trata de una patología curable.

\footnotetext{
1 Por ejemplo, Jesús Ignacio MARTÍNEZ GARCÍA, "Derecho e incertidumbre», Anuario de Filosofía del Derecho, núm. XVIII, enero, 2012; Alexandre FLÜCKIGER, «Le principe de clarté ou l'ambiguité d'un ideal», Nouveaux Cahiers du Conseil Constitutionnel, núm. 21, enero, 2007.
} 
En cambio, sabemos bien que el legislador muchas veces está naturalmente guiado, por diversas razones, a dejar un margen de maniobra a aquellos encargados de ejecutar sus prescripciones. Muchas veces acaba permitiendo a las autoridades ejecutivas un margen de poder discrecional, o bien, simplemente, como se dice en el Derecho alemán, emplea voluntariamente conceptos jurídicos indeterminados ${ }^{2}$. En todos estos casos, el sentido de la regla queda abierto y será precisado en fase de aplicación.

Aún hay más. Especialmente desde Kelsen, la teoría realista de la interpretación nos ha enseñado que, de hecho, las reglas jurídicas solo adquieren verdaderamente su sentido cuando les es dado por sus intérpretes oficiales y, sobre todo, por los jueces ${ }^{3}$. Anteriormente, incluso cuando parecen claras, solamente son portadoras de un sentido latente que necesita ser precisado. Veamos. Una regla urbanística prevé que las construcciones no deben exceder una altura de $x$ metros. Esta regla tiene un sentido tan impreciso que no se sabe desde dónde debe calcularse la altura de esas construcciones. En el Derecho francés, el juez precisó un buen día que, a falta de indicación distinta en el plan urbanístico, esta altura se calcularía no desde el punto superior de la construcción, sino desde el canalón del tejado —es decir, desde su base-: se elimina una gran incerteza, iy de una forma que posiblemente no era la que se esperaba!

Estos factores de incerteza afectan al sentido de la regla. Otros hacen peligrar sus efectos.

Como ha demostrado Hart, las reglas de reconocimiento de las normas que contienen los sistemas jurídicos concretos son a menudo vagas: fijan criterios que permiten decidir sin dudar si ciertas normas son válidas o no, aunque dejan sin aclarar la cuestión para otras reglas ${ }^{4}$.

Además, al menos en los sistemas jurídicos contemporáneos, estas reglas de reconocimiento siempre son de una complejidad considerable, lo que las hace, como mínimo, poco transparentes.

A ello habrá que añadir las grandes variaciones que afectan a la aplicación efectiva de las reglas en función de los medios empleados y del contexto. Las reflexiones contemporáneas basadas en la teoría de la regulación, sobre todo, nos han instruido definitivamente sobre estas variaciones ${ }^{5}$.

2 La distinción se debe al hecho de que el poder discrecional permite a la autoridad un margen de decisión en cuanto al contenido de su decisión, mientras que el concepto jurídico indeterminado subordina la decisión administrativa a un estándar abstracto del tipo «interés público», «bien común»... Hartmut MAURER, Droit administratif allemand, Librairie Générale de Droit et de Jurisprudence, París, 1994, pp. 127 y ss.

${ }^{3}$ Véase, por ejemplo, Isabelle PARIENTE-BUTTERLIN, Le droit, la norme et le réel, Presses Universitaires de France, París, 2005, pp. 107 y ss.; Luc WINTGENS, Droit, principes et théories. Pour un positivisme critique, Bruylant, Bruselas, 2000.

4 Véase Michel VAN DE KERCHOVE y François OST, Le système juridique entre ordre et désordre, Presses Universitaires de France, París, 1988, p. 82.

5 Jean-Bernard AUBY, «Régulations et droit administratif», Mélanges offerts à Gérard Timsit, Bruylant, Bruselas, 2005, p. 209. 


\subsection{Esto no impide tratar de reducir el grado de incerteza en un sistema y momento dados}

Incluso existe un imperativo categórico en este sentido en los sistemas que se definen como Estados de Derecho. Y no es muy difícil predecir hacia dónde deben dirigirse principalmente los esfuerzos.

a) La lucha contra la incerteza del Derecho en un Estado de Derecho es un imperativo categórico, dado que trata de garantizar en él el valor esencial de la seguridad jurídica.

Hay en la seguridad jurídica algo de estratégico para los sistemas democráticos, por dos tipos de razones.

Las primeras son de naturaleza funcional y se refieren a la eficacia misma del Derecho. Solo se puede esperar que las normas jurídicas se apliquen realmente si son comprendidos por aquellos a quienes se dirigen. A ello puede añadirse que la claridad del Derecho condiciona igualmente la confianza en el sistema jurídico, la cual también es un mecanismo para aumentar la eficacia del propio sistema ${ }^{6}$.

Las segundas razones son de orden ético y se refieren a la dignidad humana, que se respeta mejor si a los sujetos del ordenamiento jurídico se les dirigen prescripciones claras. Y esa dignidad humana no se respeta cuando se emiten órdenes cambiantes e inciertas hasta el punto de que sus destinatarios nunca saben con certeza si se están comportando como éstas prescriben.

b) En términos absolutos, los esfuerzos que pueden realizarse para disminuir la incerteza del Derecho pueden dirigirse a dos objetivos: el de la producción de normas y el de los mecanismos de aplicación e interpretación. Sobre ello volveremos más adelante, aquí nos conformamos con algunas consideraciones generales.

Si queremos mejorar la claridad de las reglas, de entrada será necesario ocuparse de la fase de producción de las normas y animar - o incluso obligar- a sus autores a ser rigurosos a la hora de dar forma al Derecho. En esto consiste la técnica normativa (légistique), es decir, la ciencia de la redacción de los textos jurídicos.

Dicho sencillamente y en pocas palabras: se trata de limitar la cantidad de reglas y afinar su formulación. Esta es una política inevitable si buscamos la claridad del Derecho, pero cuyos límites también hay que medir. La complejidad de las normas no genera necesariamente inseguridad jurídica. Todo depende del esfuerzo que deban hacer sus destinatarios para estar seguros de que se están sometiendo a ellas. La regulación de la Seguridad Social es extremadamente compleja y, sin embargo, la inserción de nuestra tarjeta de la seguridad social en el terminal del médico o del farmacéutico verifica en un abrir y cerrar de ojos la aplicación de esa regulación a nuestro caso sin que tengamos que hacer ningún esfuerzo.

${ }^{6}$ Véase, por ejemplo, Thierry TANQUEREL, Manuel de droit administratif, Schultess, Zúrich, 2011, núm. 568. 
También se puede influir en los mecanismos de aplicación e interpretación de las reglas, pues hemos visto cómo también pueden ser fuente de incerteza.

Aquí está en juego algo importante, que no es otra cosa que la imparcialidad de aquellos que son responsables de la aplicación e interpretación de las reglas ${ }^{7}$. Los prejuicios que pueden tener o los conflictos de interés en los que pueden encontrarse hacen depender sus decisiones y sus juicios de motivaciones subyacentes, a menudo imprevisibles.

Las claves que permiten protegerse de la incerteza así provocada son esencialmente de orden procedimental. La imparcialidad es un asunto procedimental, como ha demostrado Jürgen Habermas ${ }^{8}$.

\section{A PROPÓSITO DE LA EVOLUCIÓN HISTÓRICA DEL PROBLEMA}

Naturalmente, la cuestión de la incerteza del Derecho no es nueva ${ }^{9}$, y sin embargo puede demostrarse que los factores de incerteza (3.1) y los retos del problema (3.2) han evolucionado históricamente a la vez.

En la época actual, la incerteza del Derecho se alimenta de fuentes específicas relacionadas con la modificación del sentido y la función de la ley y la creciente complejidad de los sistemas normativos.

\subsection{Modificaciones del sentido y la función de la ley}

No podemos hacernos una idea de lo que actualmente genera incerteza en el Derecho sin darnos cuenta de que hay alteraciones del sentido y la función de la norma jurídica y, especialmente, de la ley.

a) Un primer fenómeno, que florece con el Estado social, es el hecho de que, si bien tradicionalmente la ley no se proponía más que establecer las reglas del juego para una vida en sociedad, en el momento actual se preocupa de dar armas a unos y otros para la dinámica social, de compensar las desigualdades y de traducir a normas las políticas económicas y sociales.

La ley ya no trata solamente de imponer comportamientos, pues también distribuye recursos para participar en la vida social. Ya no se contenta con formular grandes principios que se supone que garantizan el orden social, sino que deviene plena herramienta de las políticas públicas.

7 Stefano BERTEA, «La concezione della certezza del diritto presso alcuni interpreti del dibattito giuridico contemporaneo", Materiali per una storia della cultura giuridica, 2001, pp. 131 y ss.

8 Ibid.

9 Véase, por ejemplo, Katia WEIDENFELD, «L'incertitude du droit devant les jurisdictions parisiennes au XVo siècle», Cahiers de recherches médiévales et humanistes, núm. 7, 2000. 
Estas tendencias alimentan una producción intensiva de reglas que, por definición, genera una inestabilidad jurídica que contribuye a la incerteza del Derecho. Y alimentan igualmente la complejidad estructural de la ley, que ya no puede conformarse con órdenes simples y debe adaptarse constantemente a los cambios y evoluciones del funcionamiento social.

b) Además, estas evoluciones dieron lugar al segundo fenómeno, en cierto modo de signo inverso, que es el de la crisis regulatoria ${ }^{10}$ que se produce a partir de la década de 1980 y consiste, fundamentalmente, en un cuestionamiento de la eficacia de la legislación y reglamentación, que a menudo parecen producir más palabras que resultados concretos.

La toma de conciencia de esta realidad condujo a la vez a intentos de desregulación en esos años y a una reflexión sobre los nuevos modos de regulación, más flexibles y que se adaptan mejor al funcionamiento de la sociedad y del mercado. Concretamente, esta reflexión condujo a los tipos de regulación que hoy garantizan las autoridades administrativas independientes.

Este doble movimiento podría haber contribuido al avance a nivel de claridad y certeza del Derecho, pero en realidad no tuvo ese efecto. El movimiento de la desregulación ya se ha desvanecido y la demanda social, sobre todo de protección frente a riesgos, hace que nuestras sociedades estén cubiertas de un conjunto cada vez mayor de reglas ${ }^{11}$. En cuanto al surgimiento de nuevas formas de regulación, prácticamente no ha disminuido el nivel de incerteza del Derecho: al contrario, las decisiones de las autoridades administrativas independientes son de una complejidad especialmente grande, lo que les confiere un carácter particularmente técnico.

\subsection{Complejidad creciente de los sistemas normativos}

Esto conecta con la segunda línea de evolución poco propicia a elevar el nivel de certeza del Derecho: la creciente complejidad de nuestros sistemas normativos.

Nuestros sistemas jurídicos no solo cuentan —en mayor o menor medida— con todos los fenómenos cuantitativos de inflación normativa ${ }^{12}$, sino que también prosperan estructuralmente las fuentes de esta creciente complejidad.

Asistimos a una multiplicación de las fuentes fomentadas por la globalización y, en Europa, la europeización, incluida la aparición de un número creciente de reguladores privados. Asistimos también a una diversificación de los actores que interpre-

10 J.-B. AUBY, «Régulations et droit administratif», op. cit.

11 Por ejemplo, Jacques CHEVALLIER, L'État post-moderne, Librairie Générale de Droit et de Jurisprudence, París, 2003, pp. 99 y ss.

12 Véase Bernardo GIORGIO MATTARELLA, La trappola delle leggi, Il Mulino, Bolonia, 2011, pp. 13 y ss., y Christian MOREL, Les décisions absurdes III, Gallimard, París, 2018. 
tan normas: interpretaciones administrativas, intervención de agencias ${ }^{13} \ldots$ En este círculo ampliado, los jueces juegan un papel cada vez más estratégico ${ }^{14}$ : esto no es malo en sí, pero no reduce el grado de incerteza en el Derecho porque implica tener que esperar a la interpretación judicial para conocer el sentido de la norma legal o reglamentaria.

A ello habría que añadir también una aceleración constante del tiempo jurídico, que mantiene el flujo de producción y, por tanto, de cambio de las normas ${ }^{15}$. Y rompe el equilibrio entre normas primarias y secundarias, en el sentido que da Hart a esta distinción, con la consecuencia de que disminuye la capacidad de las secundarias para esclarecer el funcionamiento del sistema jurídico a través de la identificación de las reglas auténticamente jurídicas y, por tanto, obligatorias, a través de la adaptación de las reglas a los cambios ${ }^{16} \ldots$

Todo esto genera una complejidad que contribuye a la incerteza del Derecho. No es, como se ha dicho, que la complejidad sea necesariamente generadora de incerteza. Pero tiene ese efecto toda vez que es una complejidad no intencionada y no organizada. Este es el caso de los sistemas fragmentados como el nuestro.

\section{A PROPÓSITO DE LOS MÉTODOS DE TRATAMIENTO DEL PROBLEMA}

Vale la pena decir algo, en primer lugar, sobre las variaciones que se aprecian en la vulnerabilidad de los distintos sistemas frente a la incerteza Derecho y las razones de ser de estas variaciones (4.1). Seguidamente se discutirán los métodos más comúnmente utilizados para mejorar el grado de certeza de las reglas (4.2). Se concluirá con algunas indicaciones sobre la posición del sistema francés respecto de estas dos cuestiones (4.3).

\subsection{Las variaciones de la vulnerabilidad de los sistemas frente a la incerteza del Derecho ${ }^{17}$}

Los sistemas jurídicos son más o menos vulnerables a la incerteza de las reglas en función de factores que afectan bien a su producción, bien a su aplicación e interpretación, por retomar la distinción anterior. No se trata aquí de esbozar un panorama

13 Pierre LASCOUMES y Jean-Pierre LE BOURHIS, «Des "pase-droits” aux passes du droit. La mise en œuvre sociojuridique de l'action publique», Droit et Société, núm. 31, 1996, p. 51.

${ }_{14}$ François OST y Michel VAN DE KERCHOVE, De la pyramide au réseau? Pour une théorie dialectique du droit, Publications des facultés universitaires Saint-Louis, Bruselas, 2002, pp. 97 y ss.

15 Hartmut ROSA, Accéleration. Une critique sociale du temps, La Découverte, París, 2010, pp. 318 y ss.

16 M. VAN DE KERCHOVE y F. OST, Le système juridique entre ordre et désordre, op. cit., p. 230.

17 Para un método que permite evaluar el grado de certeza del Derecho en un sistema dado, véase Gianmarco GOMETZ, «Indici di certezza giuridica», Diritto e questioni pubbliche, 2012, p. 309. 
sistemático, lo que requeriría un estudio específico. Simplemente se dan algunos ejemplos.

a) En primer lugar, es claro que la incerteza del Derecho aumenta fácilmente por la pluralidad de fuentes internas y la complejidad de su organización. El Derecho en los Estados federales y regionales o autonómicos es, por naturaleza, más difícil de conocer con certeza, en la medida en que combina normativa estatal y local —la de los Estados federados, las regiones italianas o las comunidades autónomas españolas, por ejemplo- según una distribución de funciones que no siempre es evidente.

El grado de apertura del sistema jurídico a las fuentes internacionales es un posible germen adicional de incerteza. Los sistemas dualistas, desde este punto de vista, son naturalmente más transparentes, ya que las normas internacionales solo producen efectos si se articulan a través de leyes nacionales, mientras que, en los sistemas monistas, un tratado internacional puede colocarse junto a la ley nacional, o incluso contradecirla, sin que esto sea evidente.

También en el plano de las fuentes es claro que los Derechos jurisprudenciales son menos accesibles para el justiciable medio que aquellos que se basan en la ley escrita. $\mathrm{Al}$ justiciable medio, la combinación de normas que rigen una actividad de su interés en una ley formal, pública y relativamente estable le proporciona más certeza que su elaboración jurisprudencial, que es transparente solo para los expertos.

Fuera del sistema de fuentes, otros factores nacionales pueden jugar un papel en la determinación del nivel de certeza del Derecho en un sistema concreto. Este es el caso, por ejemplo, de las tradiciones nacionales de redacción de textos legislativos y reglamentarios. Algunas favorecen las fórmulas generales, los principios abstractos - los Derechos de la tradición romano-germánica se orientan normalmente en este sentido-, mientras otras prefieren las redacciones pragmáticas, más pegadas al problema que se regula —es la orientación habitual de los sistemas de common law-. Potencialmente, las primeras son menos propensas a proporcionar seguridad jurídica, porque los principios generales están por naturaleza abiertos a un abanico más grande de interpretaciones que las normas formuladas de forma más cercana a los problemas concretos.

b) Asimismo, en la fase de aplicación e interpretación de las normas hay diversos factores susceptibles de hacer variar el grado de certeza del Derecho en cada sistema nacional, como por ejemplo los que siguen.

Naturalmente, una cuestión esencial es la relacionada con la publicidad del Derecho, es decir, con la posibilidad que los ciudadanos tienen de estar informados, más o menos fácilmente, de la evolución del Derecho y de conocer las reglas que se aplican a su situación en un momento dado. En general, la publicidad de la ley se asegura mediante diarios oficiales. Aun así, hay que destacar que lo que esta aporta se ve contrarrestado por el hecho de que los cambios legislativos toman a menudo la forma de modificaciones de textos anteriores, cuyo alcance exacto es difícil de valorar para el ciudadano medio. 
Si bien, por lo general, la publicidad de la ley está garantizada, la de la jurisprudencia y las disposiciones administrativas más o menos internas — circulares, instrucciones, etc. - suele garantizarse de manera menos eficaz. En muchos sistemas la jurisprudencia sigue siendo confidencial: o bien rara vez se publica en abierto, o bien se difunde solamente en medios reservados para juristas.

Por lo demás, un acceso amplio a la jurisprudencia no tiene como corolario necesario un alto nivel de certeza sobre el Derecho jurisprudencial. Un elemento importante aquí es la manera en la que se redactan las sentencias. En muchos sistemas jurídicos aún se sigue haciendo de una forma muy desconcertante para el lector medio: construcciones crípticas, el uso de un lenguaje específico y, a veces, intencionadamente arcaico...

No olvidemos que el grado de certeza del Derecho también depende del modo en el que sus normas son aplicadas por las correspondientes autoridades administrativas. En ciertos sistemas, un alto grado de transparencia del funcionamiento administrativo y de fuertes garantías de imparcialidad harían más previsible la aplicación de la ley -y, por tanto, más cierto el Derecho—, mientras que en otros la tradición de secreto, la vulnerabilidad frente a conflictos de interés e incluso la corrupción hacen más aleatoria su aplicación, disminuyendo así el grado de certeza del Derecho.

\subsection{Los métodos más comúnmente utilizados para mejorar la certeza de las reglas}

Puede decirse que existe, en cierto modo, un abanico de estándares internacionales en los métodos que pueden utilizarse para reducir los factores de incerteza del Derecho, tanto en la fase de aprobación y formalización de las normas como de su aplicación e interpretación.

a) Muchos sistemas se han ocupado, algunos desde hace bastante tiempo, de mejorar la producción de las normas en la fase de su elaboración y formalización, para así luchar contra su inflación y garantizar mejor su eficacia: estas políticas tienen per se consecuencias beneficiosas sobre el grado de certeza del Derecho.

Las políticas de técnica normativa (légistique), better regulation o smart regulation se han convertido en una preocupación común en los países industrializados ${ }^{18}$. La Unión Europea es muy partidaria de ellas desde la década de 2000, lo que quedó demostrado especialmente con la Comunicación de la Comisión «Smart Regulation in the European Union» de $2010^{19}$.

18 Véase Christine PARKER, Colin SCOTT, Nicolas LACEY y John BRAITHWAITE (dirs.), Regulating Law, Oxford University Press, 2004.

19 COM (2010) 543. 
El objetivo básico de estas políticas es limitar la producción de normas. Se trata, por tanto, de políticas de simplificación ${ }^{20}$ que intentan evitar las reglamentaciones inútiles, en especial las que parecen perjudicar el crecimiento económico.

Para contribuir a frenar la inflación normativa, estas políticas ponen el foco en los mecanismos de estudio de impacto que obligan a los poderes normativos a verificar que las normas que van a aprobar tienen efectos significativos en el sentido pretendido $^{21}$.

En ciertos casos, una agencia especial se crea para supervisar la ejecución de estas políticas: un ejemplo, ya algo viejo, es el del regulatory impact officer australiano ${ }^{22}$.

Otras veces el movimiento es alentado por los jueces. Así, desde la década de 1980, el Tribunal Constitucional Federal alemán verifica si las leyes satisfacen ciertas exigencias de calidad. De la misma forma, el Tribunal de Justicia de la Unión Europea reconoce la posibilidad de verificar si los actos legislativos — nacionales o europeos- de los que conoce satisfacen el principio de seguridad jurídica ${ }^{23}$.

b) En la fase de aplicación y ejecución de las normas, los esfuerzos que pueden hacerse para reducir el nivel de incerteza del Derecho son, al menos, de tres tipos.

En primer lugar, pueden dirigirse a garantizar un mejor conocimiento de las reglas. La codificación persigue este objetivo al esforzarse en reunir las normas sobre un objeto en concreto en un marco específico y ordenado. Nunca lo consigue perfectamente, es cierto, aunque sólo sea porque las fronteras entre los códigos conllevan siempre una parte de arbitrariedad —es frecuente que una regla se refiera a dos o más sujetos que se someten a códigos distintos- y porque la legislación ulterior, en forma de modificación de los códigos, no es normalmente muy accesible para el ciudadano medio. Sea como sea, este método sigue expandiéndose: es interesante ver cómo la codificación se ha desarrollado mucho en los Estados Unidos en los últimos tiempos ${ }^{24}$.

A un nivel más modesto, la consolidación de las leyes tiene la ventaja de hacer pública la versión modificada de los textos legislativos y reglamentarios, y no únicamente su modificación.

Los esfuerzos pueden dirigirse en segundo lugar hacia el conocimiento y la comprensión de la jurisprudencia. No solo hacia su difusión, sino incluso hacia su formulación por los jueces. Podría adoptarse un sistema de formulación más directo, menos tributario del proceso al que se circunscribe la sentencia, de forma que haga

20 B. GIORGIO MATTARELLA, La trappola delle leggi, op. cit., pp. 87 y ss.

${ }_{21}$ Maria DE BENEDETTO, Mario MARTELLI y Nicoleta RANGONE, La qualità delle regole, Il Mulino, Bolonia, 2011, pp. 68 y ss.; B. GIORGIO MATTARELLA, La trappola delle leggi, op. cit., pp. 111 y ss.; Jean-Bernard AUBY y Thomas PERROUD (dirs.), Regulatory Impact Assessment, Global Law Press, Sevilla, 2013.

22 Mario CIRILLO y Julia HERTIN, Regulatory Impact Analysis in Australia, European Commission Joint Research Centre, 2004.

23 Anne MEUWESE y Patricia POPELIER, «Legal Implications of Better Regulation: A Special Issue», European Public Law, núm. 17, vol. 3, septiembre 2011, p. 455.

24 B. GIORGIO MATTARELLA, La trappola delle leggi, op. cit., pp. 169 y ss. 
más transparente la solución adoptada a la vez que los fundamentos jurídicos sobre los que descansa.

En tercer lugar, la imprevisibilidad que afecta a la aplicación administrativa de las reglas puede reducirse aumentando el nivel de transparencia de la Administración -imponiéndole, por ejemplo, la obligación de publicar los documentos internos que gobiernan su funcionamiento- y combatiendo los fenómenos de conflictos de interés y de corrupción. Sin embargo, no se trata, en el limitado ámbito de este estudio, de describir la panoplia de mecanismos a los que puede recurrirse para ello ni a los obstáculos que siempre conlleva su activación.

\subsection{Algunas indicaciones sobre la situación en el Derecho francés}

a) El Derecho francés se ha vuelto bastante sensible a las exigencias de calidad de las normas en la fase de su aprobación y formalización.

A comienzos de la década de 2000 se toma conciencia de la cuestión, al surgir informes oficiales, principalmente del Consejo de Estado, que ponen el acento sobre la proliferación legislativa y la correlativa degradación de la calidad de las leyes ${ }^{25}$.

En el mismo periodo fue la jurisprudencia, primero constitucional y luego administrativa, la que intervino en esta cuestión estableciendo un principio de claridad e inteligibilidad de la ley, cuyo ámbito de aplicación ha sido ampliado por el Consejo de Estado a los reglamentos ${ }^{26}$.

Estas variadas llamadas al orden impulsaron una política de mejora de la calidad de la ley que, desde entonces, ha destacado constantemente como una prioridad gubernamental $^{27}$.

Esto se ha traducido en estrategias de simplificación administrativa $y$ normativa ${ }^{28}$, aplicadas tradicionalmente por la Secretaría General del Gobier-

25 Destacadamente, Bruno LASSERRE, Pour une meilleure qualité de la règlementation, La Documentation Française, París, 2004; CONSEIL D’ÉTAT, Sécurité juridique et complexité du droit. Rapport public 2006, La Documentation Française, París, 2007; COUR DE CASSATION, Incertitude et sécurité juridique, 2005.

${ }^{26}$ Cons. Const., n99-421 DC, 16 décembre 1999, Codification par ordonnance, Recueil 1999, p. 136; 12 janvier 2002, n²001-455 DC, Loi de modernisation sociale; CE, 8 juillet 2005, Fédération des syndicats généraux de l'éducation nationale et de la recherche publique SGN-CFDT, n²66900; 29 octobre 2013, Association Les amis de la rade et des calanques, n³60085. Véase, por ejemplo, Anne-Laure VALEMBOIS, La constitutionnalisation de l'exigence de sécurité juridique en droit français, Librairie Générale de Droit et de Jurisprudence, París, 2015; Anne JENNEQUIN, «L'intelligibilité de la norme dans les jurisprudences du Conseil Constitutionnel et du Conseil d'État», Revue française de droit administratif, 2009, p. 913.

27 Por ejemplo, Bernard STIRN y Yann AGUILA, Droit public français et européen, Presses de Sciences Po, París, 2014, pp. 189 y ss.

28 Una visión crítica de estos esfuerzos de simplificación en Hervé MOYSAN, «Politique normative de l'État: qui trop embrasse, mal étreint?», Semaine Juridique, édition Générale, 4 de julio de 2011, p. 1331. 
no ${ }^{29}$ pero para cuya aplicación se han creado recientemente dos organismos ad hoc situados, respectivamente, bajo los ministros competentes en hacienda pública y en materia digital: la Dirección Interministerial de Reforma Pública y la Dirección Interministerial de Asuntos Digitales y del Sistema de Información y Comunicación del Estado.

En 2009 se estableció la obligación de realizar estudios de impacto sobre los proyectos de ley ${ }^{30}$. Anteriormente, en 2004, una ley ya había previsto la obligación del Gobierno de presentar un informe sobre la aplicación de cada ley transcurridos seis meses desde su entrada en vigor.

b) En el campo de la aplicación e interpretación de las normas, la historia reciente del Derecho francés se caracteriza principalmente por los siguientes elementos.

Aunque se había alejado un poco de su tradicional apego a la codificación, el sistema francés se ha vuelto a apoyar sustancialmente en ella en los últimos tiempos. Una política voluntarista para dar un nuevo impulso a la codificación comenzó con una circular del Primer Ministro de 30 de mayo de 1996 y la creación, por decreto de 12 de septiembre de 1989, de una comisión superior de codificación encargada de codificar el máximo número posible de leyes y reglamentos aún dispersos.

Desde entonces se han aprobado más de 20 códigos nuevos $^{31}$ y lo codificado abarca más del 60 por 100 de las normas con rango de ley y más de un 30 por 100 de normas reglamentarias. Entre los códigos más recientes están el código de relaciones entre el público y la Administración de 2015 — es decir, el código de procedimiento administrativo_- del que podría pensarse que habría sido elaborado mucho antes, pero que durante mucho tiempo ha chocado con la oposición de ciertas Administraciones y una cierta reticencia en el seno del Consejo de Estado.

En lo relativo a la claridad del Derecho jurisprudencial, hay que destacar el esfuerzo que hacen el Consejo de Estado y las otras jurisdicciones administrativas desde hace varios años para simplificar la redacción de sus sentencias y autos, adoptando un estilo más directo, haciendo más clara la estructura de la decisión, centrando la atención en los principios sobre los que se apoya, refiriéndose a soluciones anteriores ${ }^{32} \ldots$ La experimentación es continua.

29 Que es el órgano de relaciones entre el Primer Ministro y la Administración.

30 No se ha establecido esta obligación para los reglamentos.

31 Entre ellos, los siguientes: code de la propriété intellectuelle (1992), code de la consommation (1993), code général des collectivités territoriales (1996), code de l'environnement (2000), code monétaire et financier (2000), code de justice administrative (2000), code du patrimoine, code de l'entrée et du séjour des étrangers et du droit d'asile (2004), code général de la propriété des personnes publiques (2006), code des transports (2010) y code de la sécurité intérieure (2012).

32 Jean-Bernard AUBY, «Rédaction des jugements», Droit administratif, núm. 6, junio, 2012, ap. 6; Nathalie MASSIAS y Eric KOLBERT, «Refonte de la rédaction des décisions», La Lettre de la Justice Administrative, núm. 41, enero 2016, p. 3. 
En cuanto a la mayor imparcialidad de la Administración en la aplicación de la ley, esta ha sido objeto de diversos textos recientes sobre los conflictos de interés y la corrupción.

El primer problema ha sido tratado especialmente en la ley de 11 de octubre de 2012, sobre la transparencia en la vida pública, que impone obligaciones de declaración de patrimonio y de abstenerse de participar en ciertas decisiones a los responsables públicos susceptibles de encontrarse en un conflicto de interés.

El legislador ha vuelto recientemente sobre el segundo problema con la ley de 9 de diciembre de 2016, sobre la transparencia, la lucha contra la corrupción y la modernización de la vida económica. Este texto, que confiere un papel importante a la Agencia Francesa Anticorrupción, también ha tenido como objeto armonizar mejor el Derecho francés con las normas internacionales y europeas.

\section{CONSIDERACIONES FINALES}

La cuestión de la incereza del Derecho es una cuestión compleja, y habría sido pretencioso querer analizarla de manera profunda dentro de los límites de este texto. Por ello, no ha tenido otra ambición más que reunir algunas observaciones de carácter más bien general.

El tema, sin embargo, no es en absoluto insignificante. Existe una relación bastante evidente entre el carácter más o menos democrático de un sistema jurídico y el grado de claridad, inteligibilidad y certeza del Derecho que en él se da.

Uno de los refranes jurídicos más clásicos y conocidos dice que «la ignorancia de la ley no exime de su cumplimiento». «De acuerdo — responde el ciudadano—, ;pero a condición de que pueda saber con certeza lo que la ley me dice!».

\section{BIBLIOGRAFÍA}

Jean-Bernard AUBY, «Régulations et droit administratif», Mélanges offerts à Gérard Timsit, Bruylant, Bruselas, 2005.

— «Rédaction des jugements», Droit administratif, núm. 6, junio 2012.

Jean-Bernard AUBY y Thomas PERROUD (dirs.), Regulatory Impact Assessment, Global Law Press, Sevilla, 2013.

Stefano BERTEA, «La concezione della certezza del diritto presso alcuni interpreti del dibattito giuridico contemporaneo", Materiali per una storia della cultura giuridica, 2001.

Jacques CHEVALLIER, L'État post-moderne, Librairie Générale de Droit et de Jurisprudence, París, 2003.

Mario CIRILLO y Julia HERTIN, Regulatory Impact Analysis in Australia, European Commission Joint Research Centre, 2004.

CONSEIL D’ÉTAT, Sécurité juridique et complexité du droit. Rapport public 2006, La Documentation Française, París, 2007.

COUR DE CASSATION, Incertitude et sécurité juridique, 2005.

Maria DE BENEDETTO, Mario MARTELLI y Nicoleta RANGONE, La qualità delle regole, Il Mulino, Bolonia, 2011. 
Alexandre FLÜCKIGER, «Le principe de clarté ou l'ambiguité d'un ideal», Nouveaux Cahiers du Conseil Constitutionnel, núm. 21, enero 2007.

Bernardo GIORGIO MATTARELLA, La trappola delle leggi, Il Mulino, Bolonia, 2011.

Gianmarco GOMETZ, «Indici di certezza giuridica», Diritto e questioni pubbliche, 2012.

Anne JENNEQUIN, «Lintelligibilité de la norme dans les jurisprudences du Conseil Constitutionnel et du Conseil d'État", Revue française de droit administratif, 2009.

Pierre LASCOUMES y Jean-Pierre LE BOURHIS, «Des "pase-droits" aux passes du droit. La mise en œuvre sociojuridique de l'action publique», Droit et Société, núm. 31, 1996.

Bruno LASSERRE, Pour une meilleure qualité de la règlementation, La Documentation Française, París, 2004.

Jesús Ignacio MARTÍNEZ GARCÍA, "Derecho e incertidumbre», Anuario de Filosofía del Derecho, núm. XVIII, enero 2012.

Nathalie MASSIAS y Eric KOLBERT, «Refonte de la rédaction des décisions», La Lettre de la Justice Administrative, núm. 41, enero 2016.

Hartmut MAURER, Droit administratif allemand, Librairie Générale de Droit et de Jurisprudence, París, 1994.

Anne MEUWESE y Patricia POPELIER, «Legal Implications of Better Regulation: A Special Issue», European Public Law, núm. 17, vol. 3, septiembre 2011, p. 455.

Christian MOREL, Les décisions absurdes III, Gallimard, París, 2018.

Hervé MOYSAN, «Politique normative de l'État: qui trop embrasse, mal étreint?», Semaine Juridique, édition Générale, 4 de julio de 2011.

François OST y Michel VAN DE KERCHOVE, De la pyramide au réseau? Pour une théorie dialectique $d u$ droit, Publications des facultés universitaires Saint-Louis, Bruselas, 2002.

Isabelle PARIENTE-BUTTERLIN, Le droit, la norme et le réel, Presses Universitaires de France, París, 2005.

Christine PARKER, Colin SCOTT, Nicolas LACEY y John BRAITHWAITE (dirs.), Regulating Law, Oxford University Press, 2004.

Hartmut ROSA, Accéleration. Une critique sociale du temps, La Découverte, París, 2010.

Bernard STIRN y Yann AGUILA, Droit public français et européen, Presses de Sciences Po, París, 2014. Thierry TANQUEREL, Manuel de droit administratif, Schultess, Zúrich, 2011, núm. 568.

Anne-Laure VALEMBOIS, La constitutionnalisation de l'exigence de sécurité juridique en droit français, Librairie Générale de Droit et de Jurisprudence, París, 2015.

Michel VAN DE KERCHOVE y François OST, Le système juridique entre ordre et désordre, Presses Universitaires de France, París, 1988.

Katia WEIDENFELD, «L'incertitude du droit devant les jurisdictions parisiennes au XVo siècle», $C a$ hiers de recherches médiévales et humanistes, núm. 7, 2000.

Luc WINTGENS, Droit, principes et théories. Pour un positivisme critique, Bruylant, Bruselas, 2000. 
